\title{
Assessing the energy impact of traffic management and vehicle hybridisation
}

\section{Dominik Karbowski, Namwook Kim and Joshua Auld}

Center for Transportation Research, Argonne National Laboratory,

Lemont, IL, USA

Email: dkarbowski@anl.gov

Email: nakimanl.gov

Email: jauld@anl.gov

\section{Vadim Sokolov*}

George Mason University,

Nguyen Engineering Building 2242,

Fairfax, VA, 22302, USA

Email: vsokolov@gmu.edu

*Corresponding author

\begin{abstract}
We provide a review of methodologies previously used to evaluate impacts of transportation systems and changes in transportation infrastructure on energy consumption. We present a new framework that allows estimating the energy impacts of managed traffic lanes in the context of vehicle automation. The presented framework relies on two major components, an integrated transportation system simulator and a powertrain simulator. For the transportation system simulator we propose using integrated transportation system simulator POLARIS. For the powertrain simulator we use AUTONOMIE, a tool funded by the US Department of Energy. Both tools are developed at Argonne National Laboratory. We demonstrate our approach by modelling a transportation corridor along a major highway. Two scenarios are considered, unmanaged, when both trucks and cars use all the lanes of the highway and managed, under which one of the highway lanes is a dedicated lane for truck traffic and trucks are forming platoons using adaptive cruise control technology. We provide the numerical results of the experiment at the end of the paper. We also present the impact of vehicle hybridisation combined with automation on the energy consumption.
\end{abstract}

Keywords: travel demand; traffic microsimulation; energy assessment; integrated modelling; powertrain simulation.

Reference to this paper should be made as follows: Karbowski, D., Kim, N., Auld, J. and Sokolov, V. (2016) 'Assessing the energy impact of traffic management and vehicle hybridisation', Int. J. Complexity in Applied Science and Technology, Vol. 1, No. 1, pp.107-124. 
Biographical notes: Vadim Sokolov is an Assistant Professor in the Systems Engineering and Operations Research Department at George Mason University. He works on building robust solutions for large scale complex system analysis, at the interface of simulation-based modelling and statistics. This involves, developing new methodologies that rely on agent-based modelling, Bayesian analysis of time series data, design of computational experiments and development of open-source software that implements those methodologies. Inspired by an interest in urban systems he co-developed mobility simulator called Polaris that is currently used for large scale transportation networks analysis by both local and federal governments.

Dominik Karbowski received his Master's degree in Science and Executive Engineering with a major in energy systems from Mines ParisTech, France in 2006. At Argonne, he works on modelling advanced powertrains such as hybrids or plug-in hybrids, both light- and heavy-duty. He focuses his research on vehicle level control optimisation and powertrain design optimisation.

Namwook Kim is an Assistant Professor at Department of Mechanical Engineering Hanyang University. His research area is in developing, validating, and evaluating simulation models for advanced vehicles. Dynamics, optimisation, and optimal control theory are vigorously used for these studies, and optimal control for hybrid electric vehicles was the theme of his dissertation.

Joshua Auld is a Computational Transportation Engineer at the Argonne National Laboratory Transportation Research Systems Modelling and Control Group in the Energy Systems division. He received his Master's degree in May 2007 and doctorate in August 2011 from the Civil and Materials Engineering Department at the University of Illinois, Chicago with a concentration in transportation. He also received his post-doctoral appointment with the University of Illinois at Chicago and Argonne's Transportation Research and Analysis Computing Center in December 2014.

\section{Introduction}

A transportation system comprises the regional road network, the transit network as well as the users of the system: drivers and passengers. Research on transportation systems analyses how the users move throughout the network, and the interactions between demand (users) and supply (the network). Transportation system models allow policy makers to forecast the impacts of new policies or new facilities on the performance of a transportation system. Usual performance metrics for a transportation system are time delays (level of service) and emissions associated with congestion. Energy consumption considerations are on the other hand rarely incorporated into the traditional decision-making process.

Most of the users of the network move around using a vehicle: motorbike, car, bus, truck, train, etc. Those vehicles consume energy and contribute to harmful emissions. As a result there is a strong push to develop and introduce new technologies that would lead to cleaner vehicles. Research in that field often relies on powertrain models; they allow to predict the energy consumed by a vehicle with any given combination of technologies 
under different conditions and for different drive cycles. It is therefore possible to predict the energy efficiency of a vehicle well before it is actually built.

Most of the research in the area of transportation systems modelling is targeted on reducing congestion and improving travel time. Similarly, researchers in vehicle systems focus on improving energy efficiency on standardised cycles. We build on existing modelling techniques to develop a high fidelity to assess global energy performance in a real-world transportation network scenario. The proposed integrated framework would bridge the gap between powertrain simulation and transportation system simulation and would greatly help to quantify the relationship between the way the network is used and the energy consumed by vehicles in it.

We describe a new software framework that allows to estimate energy consumption not by a single vehicle but rather the entire transportation system. In such integrated model, the vehicle would be modelled in the context of an urban region and its transportation network with congestion, traffic signals, stop signs, alternative transportation modes, etc. Concretely, the framework includes a microscopic traffic and demand model, and will compute the energy consumption of every trip.

\section{Energy consumption of a transportation system}

A typical approach to estimating energy consumption and environmental impacts of a transportation system is to combine a model that forecasts traffic flows given a scenario and another model that estimates emissions and energy given the output of traffic flow forecasts. This approach allows to estimate benefits of new transportation policies, new management strategies or newly build infrastructure. A high-fidelity energy model is desirable so that it is possible to design and evaluate new scenarios, while having a high level of confidence that savings observed in simulation will be matched in the real-world implementation. The model should also take the future evolutions of vehicle technologies and energy sources for transportation in order to represent a probable future vehicle fleet. For example, it is expected that battery-electric vehicles (BEVs) and electrified vehicles will have a non-negligible market share in the next 15 years. We review several important factors that can impact energy consumption in transportation.

\subsection{Factors impacting energy consumption in transportation}

Fuel economy regulations and environmental concerns have had the automotive industry and research community propose and introduce advanced fuel saving technology, as well as alternative energy power trains.

Each of the 'traditional' components have significantly evolved and improved. Modern engines are more efficient, cleaner, smaller and lighter thanks to the widespread use of turbocharging, advanced fuel spray designs and complex controls (e.g., VVT-VVL, GDI, advanced fuel cut-off). Ancillary losses have also been reduced, especially through electrification and better control. Modern transmissions are more efficient thanks to a higher number of gears (up to eight gears), better controls (early torque converter lock-up) or alternative designs (CVT, DCT). Chassis are getting more aerodynamic thanks to optimised vehicle shapes, improved cooling air path and aerodynamic add-ons (e.g., active shutter grilles), while tires have reduced rolling 
resistance. Lightweighting has affected the entire vehicle thanks to the use of lighter materials in conjunction with advanced structures (e.g., aluminium chassis). All these improvements have not been used solely towards fuel consumption reduction; often they help the manufacturer propose a better trade-off between in the fuel economy/performance/features space.

In parallel, system design optimisation in conjunction with complex energy management allows to save more energy at the system level. The major evolution in the past 15 years has been the emergence and now more and more widespread use of hybrid electric powertrains (HEV). The benefits of hybridisation and electrification are maximised with a smart management of the various components onboard.

Finally, constant breakthroughs in battery, power electronics and electric machine design and manufacturing will enable more BEVs and plug-in HEVs (PHEV) to hit the road. The energy model has thus to take into account all these new trends; the risk of not doing so may lead to vastly inaccurate predictions, and pick the wrong scenarios as optimum.

Another factor is vehicle operations. We define here macroscopic factors the ones that are due to the way vehicles are used (or not used). They are independent of the vehicle energy efficiency. They are also the only factors that can be influenced as part of an infrastructure or system management project, as fleet composition and vehicle technologies are out of the scope of the project. Below is a list of macroscopic factors.

- Vehicle trip demand: whether a vehicle travels or not has the biggest impact on energy use. Putting a vehicle off the road can be done through activity shift (e.g., telecommuting), vehicle sharing (transit, carpooling, car-share, taxi), shift to non-motor vehicles (biking), or a combinations of all of them. Attention must be paid to properly estimating the impact of vehicle sharing because its inefficient use (e.g., low ridership buses) may have negative energy consequences.

- Vehicle travelled distance is the second biggest driver of energy consumption. Reducing distance of travelled can be achieved through better trip planning (e.g., picking closer alternative destinations with same utility) or better routing in the case of multiple destinations. Eco-routing, which consists in choosing the most energy efficient route, is often associated with reducing travel distance, although it has to take into account the traffic, intersections, speed limits and topography as well.

- Vehicle driving operations: the way vehicles are driven on a particular distance significantly impacts energy consumption because vehicles energy efficiency depends on the operating points or because some the physical consequences of certain behaviours, such as drag force due to high speed, are unavoidable. However, it is important to note that differences in driving operations do not affect all vehicles the same way, e.g., removing traffic stop results in more savings for a conventional ICE-powered than for a hybrid car which can recuperate some of the braking energy and avoid engine idling at the stop. Driving operations can be operator-specific (aggressive driving vs. calmer driving) or related to the network configuration and operations (intersections, speed limits, congestion, accidents, etc.). Making driving more energy efficient therefore requires: driver coaching, better infrastructure control or better trip planning (travelling at off-peak hours, selecting routes with smoother traffic). Driving operations are also critical for the high topography: when going uphill, energy must be spent to increase the potential energy of the vehicle; 
part of that energy can be recuperated when going downhill. Topography must be integrated in any 'green routing' algorithm, because it can significantly affect energy consumption.

\subsection{Energy modelling in transportation}

There are numerous examples of predicting the energy use of vehicles in modelled traffic situations. In most cases, the goal is to model emissions in general, harmful pollutants ones in particular $\left(\mathrm{CO}, \mathrm{HC}, \mathrm{NO}_{\mathrm{x}}\right.$, etc.). $\mathrm{CO}_{2}$ is also often included, and it is directly related to fuel use (these examples only look at gasoline/diesel vehicles). These studies generally involve linking a traffic simulation tool with an emission model.

To model the emissions at the scale of an entire area, it is necessary to use a macroscopic traffic model. In Europe, the emission models HBEFA and COPERT are used. These two models were compared for the City of Madrid (Borge et al., 2012). HBEFA is also listed as a plug-in for the commercial tool PTV VISSUM (PTV Group, n.d.). There are examples of its use with MATSim (Balmer et al., 2008) for the City of Munich (Hülsmann et al., 2014). In North America, there are examples of integration of the EMME traffic simulation tool (Babin et al., 1981) with US EPA's emission model MOBILE (Armstrong and Khan, 2004; Hatzopoulou and Miller, 2010), which development ceased in the early 2000s. There are numerous studies focusing on specific areas of a transportation network, such as major highways, small neighbourhoods or arterial roads. They rely on microscopic traffic flow simulators, which can output naturalistic second-by-second speed profiles for each travelling vehicle in the area of interest, which then can be used as inputs to more complex vehicle energy/emission models. PTV VISSIM lists ENviVer (based on VERSIT+) as a plug-in for emission and energy modelling (PTV Group, n.d.), but there are also examples of ad-hoc linkages with MOVES (Abou-Senna and Radwan, 2013), PHEM (Hirschmann et al., 2010) and CMEM (Stevanovic et al., 2009). Quadstone Paramics, another commercial microsimulation tool has been used in conjunction with VERSIT+ (Madireddy et al., 2011), MOVES (Xie et al., 2012) and CMEM (Boriboonsomsin and Barth, 2008; Misra et al., 2013). CMEM was also used in a study of Lee et al. (2009), while the energy model VT-micro was used with TRANSIMS (Kwak et al., 2012). Sokolov et al. (2014) have used a mixedscale approach: a mesoscopic traffic simulation tool, POLARIS was used to generate travel demands, subsequently modelled at a microscopic level with VISSIM. The resulting speed profiles were then fed into a high-fidelity energy model, Autonomie.

To predict the energy use of a particular vehicle travelling a particular trip, the vehicle energy model is designed. A review of the main modelling approaches as well as particular implementations is presented in hereinafter.

The most elementary model for estimating energy use is the single distance-specific energy consumption figure ('MPG sticker'), often given by the manufacturer as part of a certification procedure, supervised by the US EPA in the USA. While implementation is straightforward data for current and past vehicles is easily available, this model is excessively simple and is only sensitive to distance; it does not take into account the diversity of driving, so traffic or eco-driving have no impact. It is also limited to existing light-duty vehicles and does not allow to model future vehicles. As a result it is not for scenario comparison and optimisation. Examples include Pendyala (2014). 
In this type of model, the trip is divided into 'bins' of uniform driving patterns, e.g., congested highway) using one or more variables as a statistical estimator of the state (e.g., average speed). For each vehicle modelled, there is one energy consumption value for each bin, per unit of distance. The total energy for a particular trip is the weighted sum each bin energy consumption weighted by the distance travelled in each bin. The model is populated from real-world measurements or from a more detailed physical model. There are several types of binning models, based on the type of bin used. For 'average speed binning models', the only variable is the average speed. Implementations include MOBILE, developed by the US EPA but replaced by MOVES in the mid-2000 (US EPA, 2014). A similar model is EMFAC (California Air Resource Board, 2011). In the EU, COPERT includes fuel consumption estimates for various vehicle categories and for three types of driving (rural, urban, motorway) as a function of average vehicle speed (http://emisia.com/copert; Ntziachristos et al., 2009; Zachariadis and Samaras, 1999). In 'traffic-situation binning models', the driving situation is identified by multiple variables. For example HBEFA (INFRAS) uses area type, road type, speed limit and level of service. Energy consumption/ $\mathrm{CO}_{2}$ emission factors are obtained from a physical model, PHEM (Hausberger et al., 2009). 'Microscopic binning models' require the knowledge of the speed profile with greater temporal resolution. In particular, they rely on average values of acceleration (or equivalent parameter). Earlier versions of VERSIT+ (Smit et al., 2007) use acceleration and speed as their main variables. MOVES (US EPA, 2014), first released in 2004, uses speed and vehicle specific power (VSP), which is road load per unit of mass and distance, and data comes from uses a combinations of realworld measurements and the physical model PERE to 'fill the gaps'. The fuel consumption has been updated since 2004 only by multiplication ratios from CAFE. The models with lower resolution ('average speed' and 'traffic situation') are well suited to work with microscopic or mesoscopic traffic simulation tools, while the 'microscopic' are better suited to microscopic traffic model. The quality of their predictions relies on the quality of the data used to create them (measurements of model). Also the relatively simple nature of these statistical models does not allow them to capture all the complexities of advanced technologies and complex vehicle control, electrified vehicles in particular.

In these models, the instantaneous fuel rate is a polynomial function of speed and acceleration, with some refinements in each implementation, and coefficient identification by matching total fuel consumption for entire trips (such as certification cycles) or large trip sections. For example, the Virginia Tech model VT-CPFM (Rakha et al., 2011), preceded by VT-micro first estimate vehicle specific power (VSP) based on speed and acceleration and the polynomial variable is VSP or VSP and engine speed (which is related to vehicle speed). Only three coefficients per vehicle are needed, which is likely to be too simplistic to provide more than rough estimations. In the later versions of VERSIT+ (Ligterink and de Lange, 2009), the variable used is w a linear combination of speed and acceleration, and the polynomial have different definitions based on the speed classes and the sign of $\mathrm{w}$, resulting in a total of nine coefficients, which probably leads to a better model. Curve-fit models are not well suited for vehicles with advanced controls, such as HEVs because instantaneous fuel rate depends on the previous operating states of the vehicle (for example battery state-of-charge). Backward-looking microscopic model: these physical models combine the estimation of the vehicle road load and a simple model of the powertrain. They require a microscopic speed profile (1 Hz or more). The road load takes into account the aerodynamic drag, the rolling resistance, 
acceleration and road slope. Assuming a transmission ratio using a shifting logic, transmission losses and ancillary load, the engine torque and speed are computed. Fuel consumption is then estimated, often from a simple physical model of the engine, though better models use fuel rate maps from engine tests. These models can be relatively accurate if the components are appropriately modelled, but quickly show limitations when the powertrain is more advanced and when the vehicle control is key to the fuel savings, such as HEVs. In this case a forward-looking approach is preferred. There are two main classes of models. Spreadsheet models that are implemented in Excel; thus, limiting their level of complexity and of computational efficiency. Examples include PERE (Nam, 2004), originally developed by the US EPA to fill the gaps in MOVES but not updated since; FASTSIM (Brooker et al., 2015), recently developed at the National Renewable Energy Laboratory (NREL). Coded models are implemented using a coding language, often in Matlab and allows more refinements in the models as well as better modularity. Examples include PHEM (Hausberger et al., 2009) developed at TU Graz for computing emissions for HBEFA; the comprehensive modal emissions model (CMEM) (Barth et al., 1996).

These models mimic the 'driver and chassis dynamometer' situation where a driver follows a predefined second-by-second speed profile. The model is called forward-looking because commands produce efforts (torques, forces, etc.) which results in flows (speed). It therefore models more accurately the causality of the real-world. Because of that, they are also a better choice for modelling complex control strategies and complex powertrains. Such models are the type of models used by the automotive industry for energy consumption and performance. Examples include GT-Drive (Ciesla et al., 2000; http://www.gtisoft.com/products/GT-SUITE_Overview.php), AVL cruise (https://www.avl.com/cruise/), Autonomie (http://www.autonomie.net/), GEM (https://www3.epa.gov/otaq/climate/gem.htm), ALPHA (https://www3.epa.gov/otaq/ climate/alpha.htm).

\section{Integrated transportation system simulation}

To model an urban transportation system using an agent-based approach, each traveller is autonomous and makes decisions based on the needs, information available on the current transportation system state and historic data. Our model builds on the previous work, in particular models such MITSIMLab (Yang et al., 2000), DynaMIT (Ben-Akiva et al., 2002) and Dynasmart (Mahmassani et al., 1993), with the addition of a disaggregate model for simulating individual activity-travel behaviour. Our model consists of an activity-based demand model (ABM) implemented as a series of actions and behaviours that the traveller agents perform during the simulation, following the activity-based paradigm. Along with the ABM is the network model, which includes an individual traveller route choice model under information, a route generation model using simulated travel costs, and a traffic simulation based on the kinematic wave theory of traffic flow. Finally, there is a traffic management centre (TMC) component which controls the ITS system. The innovative aspect of this model is the complete integration of the activity-based and network simulation model elements through their implementation as behaviours of a single, persistent traveller agent which interacts directly with the network and ITS agents in a shared memory space. This work builds on 
previous integration efforts such as MATSIM (Balmer et al., 2008), SimTRAVEL (Pendyala et al., 2012), and the DaySim-TRANSIMS integration (Lawe et al., 2011). The activity-based model consists of a series of agent classes which implement events corresponding to typical components found in travel demand, network simulation and operations models. At the centre of the model is a person-agent which represents the travellers in the system and their activity and travel planning behaviour. The person agents operate in an environment represented by the transportation network agents to handle movements through the system. A set of ITS components and an automated TMC agent controls the ITS system and monitors the network agents. An overview of the ABM is shown in Figure 1, which shows how the main actions and behaviours of the three main components of the model and how they fit together. The various components are discussed in the following sections, followed by a section which describes the case studies carried out using the model. Throughout these sections, it is important to note that both the simulation model itself and the Chicago-area network event case studies are currently only intended as demonstration prototypes.

Figure 1 POLARIS overview (see online version for colours)

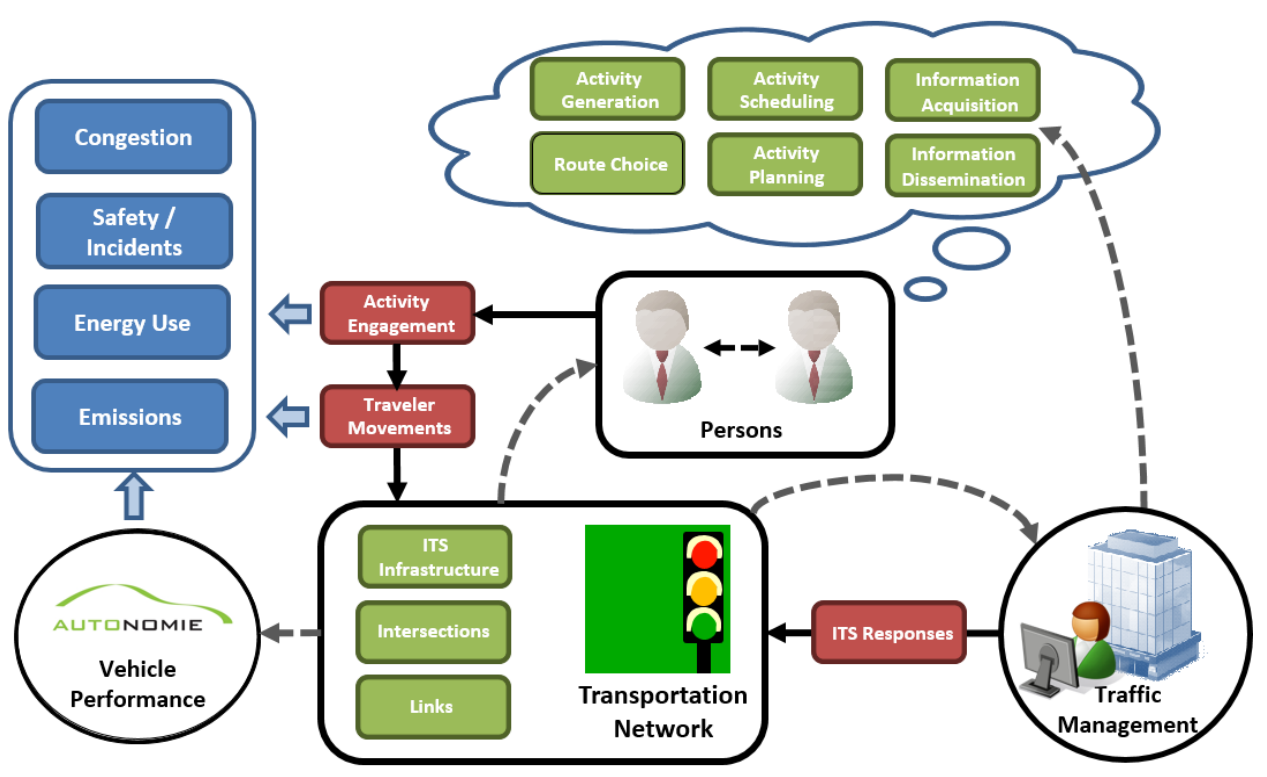

\section{Activity-based travel demand modelling}

The foundation of the integrated transportation simulation system is the activity-based demand modelling components which represent individual traveller behaviour. The modelled behaviours include such aspects as activity generation, activity attribute planning and replanning, and activity scheduling, rescheduling and conflict resolution. The behaviours are all implemented to be responsive to network events and traffic management through agent replanning models. The behavioural models in POLARIS are based on the agent-based dynamic activity planning and travel scheduling (ADAPTS) model which was designed as a simulator of the individual activity and travel planning 
and scheduling process (Auld and Mohammadian, 2009). The model was designed with the goal of continuously integrating travel demand modelling and dynamic traffic simulation with the idea that considering planning and scheduling steps as discrete events within an overall simulation, a more consistent picture of traveller behaviour is developed. The key aspect of the model is that activity planning/replanning and scheduling occur continuously throughout the simulation along with the trip routing and traffic simulation. In other words an individual agent's experience in the network simulation constantly informs the need to plan later activities or replan the current activity/trip, resulting in a consistent schedule that is responsive to network dynamics.

The ADAPTS activity-based model has been reimplemented in the POLARIS framework as a series of individual agent behaviours which perform the planning and scheduling. The main traveller behaviours that are implemented include: activity generation, activity planning and replanning, activity-travel scheduling and travelling. The activity generation behaviour of an agent is implemented as a competing hazard-duration model which periodically estimates the need for generating new activities as the simulation progresses based on the times since each activity type was last performed, see Auld et al. (2011). The attributes of each generated activities are then planned at times determined by a series of linked multivariate ordered probit regression models described in Auld and Mohammadian (2012), collectively referred to as the activity planning process models. The choice behaviours for each aspect of the activity plan include destination choice and mode choice, which are implemented as discrete choice models, and start time and duration which are drawn from observed probability distributions. After planning, the activities are scheduled using a heuristic scheduling rule base along with a decision-tree conflict resolution model, where the conflict resolution model determines the overall strategy to implement in resolving scheduling inconsistencies and the scheduling rules implement the strategy while maintaining a consistent activity-travel schedule. The scheduling process occurs whenever a new activity is generated and planned, an activity is re-planned for any reason, or deviations from the original travel schedule are created through traffic simulation. Finally, the travelling behaviours of the individual included routing/rerouting and movement. When an activity start point in the individual schedule is reached during simulation the person plans a route using an individualised router. The routed trajectory is then loaded to the network. It is important to note here that the person agent is truly a persistent agent, i.e., while the person is moved by the network simulation all aspects of that agent remain attached to the agent, such as demographic characteristics, history, schedule and so on, and the agent can continue to implement its individual planning and scheduling behaviours.

\section{Transportation network model (supply)}

The network model components include three key models: an individual route choice model, a route generation model using simulated travel costs, and a mesoscopic traffic simulation model. Our framework allows seamless integration of network and demand models. In our current implementation, prevailing traffic information is incorporated in pre-trip and/or en-route choices. When choosing a route, algorithms accounts for agent's own user characteristics and incorporates current congestion levels when calculating 
minimal cost path on the network. Currently, simulated travel times are used as costs. However, model can easily incorporate other metrics, such as pricing, energy and exposure to air pollution. Each traveller has its own copy of the network topology and costs, which enables the use of heterogeneous sources of traffic information such as historical, prevailing, experienced and predictive travel costs for different user classes. The router uses the A-star shortest path algorithm, which allows the parallelisation of route calculation by each vehicle. It is also consistent with the integration with activity-based demand model to calculate routes between two activity locations instead of two traffic analysis zones. Our framework allows to model dynamic route choice decisions under various information provision types. The purpose of the route choice model is to address en-route switching behaviour of travellers. Currently the model distinguishes several types of travellers, those with pre-trip traffic information only, and equipped travellers with en-route information (e.g., navigation devices). Pre-trip decisions for both traveller types are based on the prevailing traffic conditions. In addition, the equipped travellers can access real-time traffic information while en-route. The unequipped travellers can, however, access real time traffic information disseminated through ITS infrastructures such as variable message signs and radio to respond to both recurrent and non-recurring traffic congestions. A bounded rationality en-route switching model (Jayakrishnan et al., 1994; Mahmassani and Stephan, 1988) is used.

Once the pre-trip choices on route, destination and departure time were assigned to agent, traffic flow simulator is used to model congestion level of road network. Kinematic wave theory of traffic flow (Newell, 1993) is used to simulate traffic flow on each road segment. This model has been recognised as an efficient and effective method for large-scale networks (Lu et al., 2013) and dynamic traffic assignment formulations. In particular, the Lighthill-Whitham-Richards (LWR) model (Lighthill and Whitham, 1955; Richards, 1956) along with discretisation scheme proposed by Newell (1993) is used. In addition, intersection operations are simulated for signal controls, as well as stop and yield signs. The input for LWR model is so called-fundamental diagram that relates flow and density of traffic flow on a road segment. The key parameter of fundamental diagram is critical flow, which is also called road capacity. The capacity is measured in number of vehicles per hour per lane, and provides theoretical maximum of flow that can be accommodated by a road segment. One of the important features of our traffic flow model is sensitivity to non-recurrent events, such as weather or road accidents. The response of road network to such events is modelled by reducing capacity using a scaling factor for each of the event types.

\section{Powertrain simulation}

The vehicles are simulated in Autonomie (http://www.autonomie.net/), which was developed by Argonne National Laboratory (Figure 2). It is the main vehicle simulation tool used in the US Department of Energy's FreedomCAR and Vehicle Technologies Program. It has been used in numerous studies to give the US Government guidance for future research. More than 140 companies and research entities, including major automotive companies and suppliers, also use Autonomie to support advanced vehicle development programs. Autonomie is a software package with a user-friendly interface that allows fast selection of powertrain configurations and component models, initialisation data and processing files. It includes a wide range of pre-defined vehicles, 
both light and heavy-duty. All models and calculations are based on Matlab and Simulink: a vehicle is built in Simulink based on the selections made in the interface, and it is similarly initialised.

The resulting vehicle model is a forward-looking model: a driver model sends a pedal signal to the supervisory control of the vehicle, which in turn sends a torque demand to the engine and other power sources. The components use an effort/flow topology, inspired by the Bond graph theory: an effort (e.g., torque) is propagated forward until it is integrated to compute a flow (e.g., speed) which is itself propagated backwards. The plant models (engine, transmission, etc.) are generally map-based, combining accuracy and short execution time. Vehicles and individual components were validated based on test data.

Figure 2 Autonomie GUI (see online version for colours)

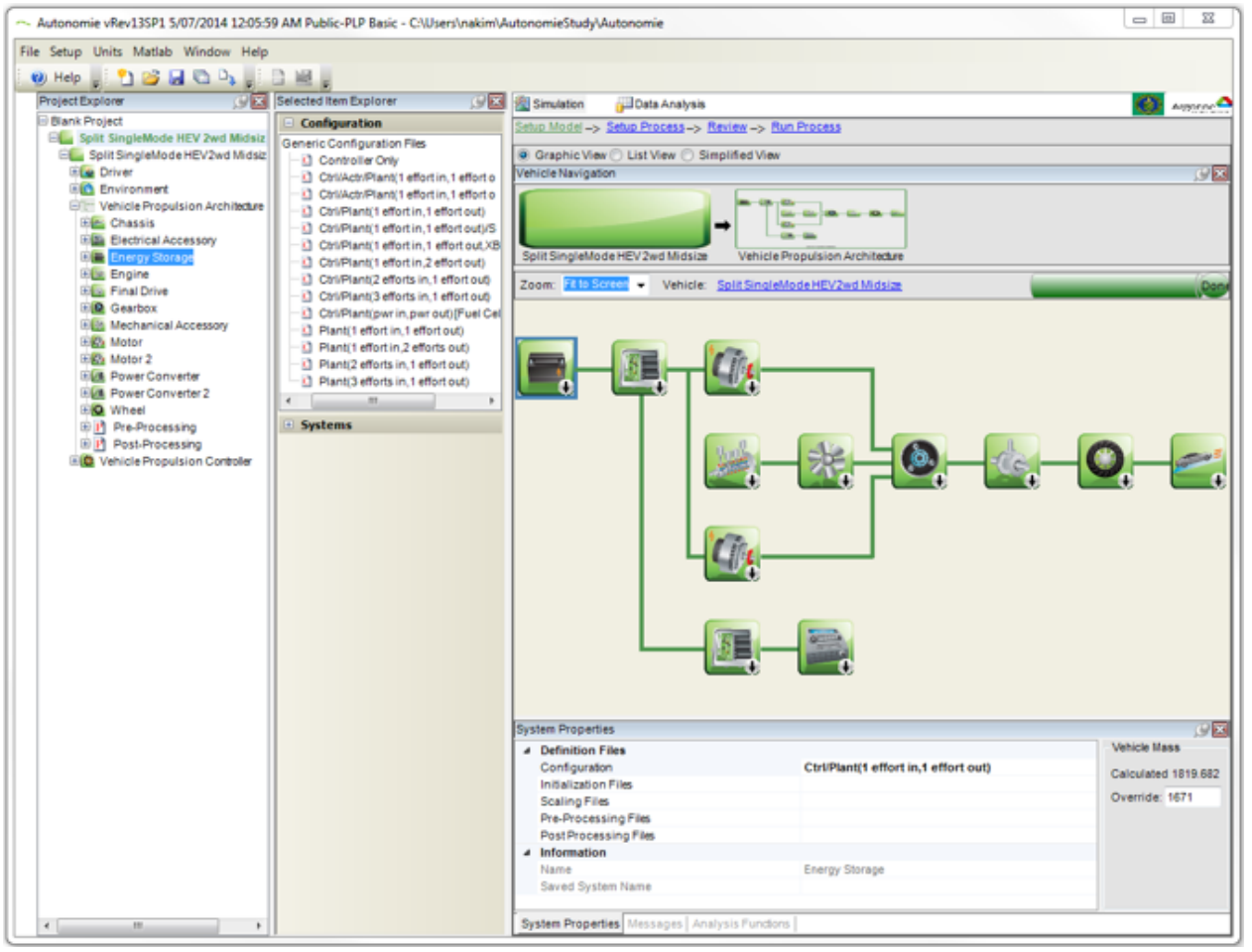

\section{Energy intensity impacts assessment case study}

The output of the integrated transportation system model is the trajectories of the vehicles on the transportation network. Those trajectories are results of an evolved behaviour of agents in a complex system. The decision made by agents on travel activities, routes, departure times are effected by other agents and the management strategies implemented through the ITS infrastructure (VSS, VMS, HAR, etc.) The next step is to assess the energy impact of specific management strategy. In our framework we propose using 
AUTONOMIE simulator that allows to accurately estimate the energy consumed by a vehicle provided the speed profile. However, the speed profile generated by POLARIS traffic simulator component is not of low enough time resolution and thus cannot be used by AUTONOMIE as is. We propose to use a microscopic traffic simulator for a small subarea of a regional network. Unfortunately none of the existing microscopic traffic simulators are capable of simulating an entire metropolitan area due to computational requirement. Another aspect that prohibits creating a regional scale microscopic simulation model is the amount of data needed to build and calibrate the model. Currently we are in the process of integrating the AUTONMIE simulator and large scale transportation system simulator.

Figure 3 Overview of the proposed framework (see online version for colours)

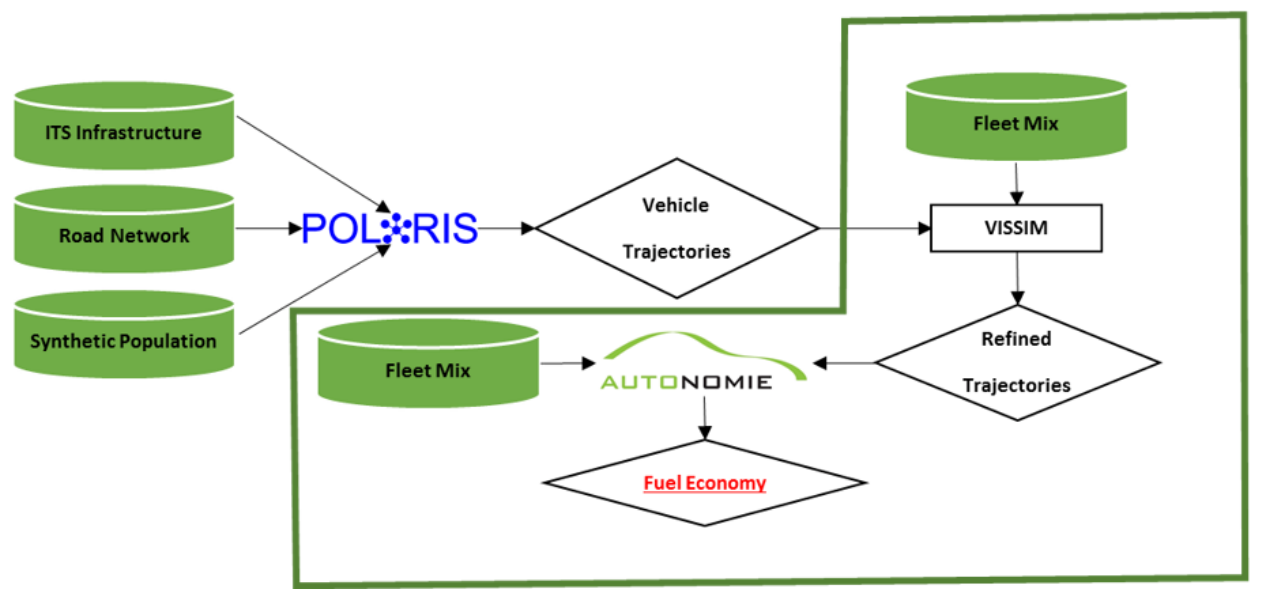

For the purposes of demonstrating a prototype we considered a small portion of a network and integrated VISSIM with the AUTONOMIE only (the green area on Figure 3). We used a stretch of a 4-lane highway, $19 \mathrm{~km}$ long with several on and off ramps. We considered mixed traffic with two vehicle classes: light-duty (CAR) and heavy-duty (HDV) vehicles, with the shares of each type are shown in Figure 4.

Figure 4 Vehicle class composition for all scenarios (see online version for colours)

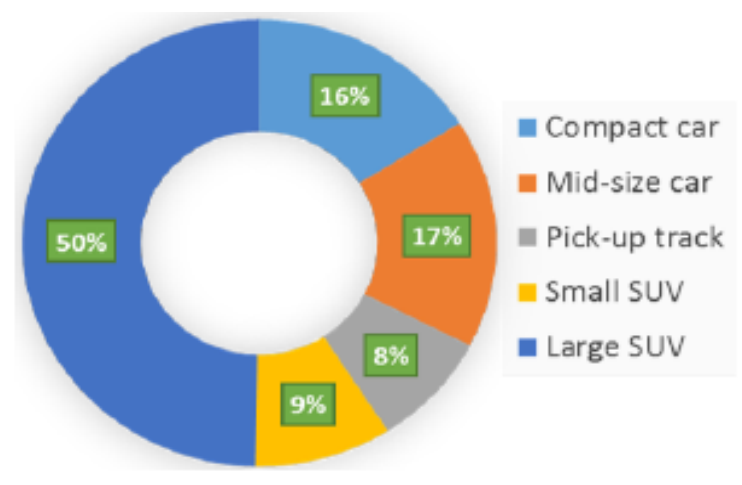


We considered three fleet scenarios that correspond to different percentages of hybrid electric vehicles in the traffic flow as shown in Table 1.

Table 1 Vehicle fleet scenarios with different percentages of HEV present in the traffic flow

\begin{tabular}{lcc}
\hline Fleet scenario & Light-duty & Heavy-duty \\
\hline FS1 (Baseline) & $3 \% \mathrm{HEV}$ & $0 \% \mathrm{HEV}$ \\
FS2 (HEV low) & $10 \% \mathrm{HEV}$ & $10 \% \mathrm{HEV}$ \\
FS3 (HEV high) & $10 \% \mathrm{HEV}$ & $20 \% \mathrm{HEV}$ \\
\hline
\end{tabular}

Both CAR and HDV classes has a conventional ICE version (CV) and a hybrid (HEV) version. Furthermore, each vehicle template has a unique combination of components and average mass. We considered two strategies managed and unmanaged. In unmanaged scenario, both vehicle types were allowed to use all of the lanes. In managed scenario the left-most lane was a dedicated lane for truck traffic. For the managed scenario we considered two cases, the first, when trucks form platoons using automated cruise control (ACC) and the second, when they do not. The vehicle characteristics used for the simulation, including average mass, are shown in Table 2.

Table 2 Vehicle specifications

\begin{tabular}{lcc}
\hline Vehicle type & CAR (midsize conv.) & HDV (heavy duty conv.) \\
\hline Mass mean & $1,500 \mathrm{~kg}$ & $14,900 \mathrm{~kg}$ \\
Powertrain & 5 gear automatic & 6 gear automatic \\
Engine & $149 \mathrm{~kW}$ & $243 \mathrm{~kW}$ \\
\hline
\end{tabular}

Table 3 shows the result for estimated summary statistics for speed and acceleration.

Table 3 Speed and acceleration summary statistics

\begin{tabular}{llcccccc}
\hline & & Min. & 1st Qu. & Median & Mean & 3rd Qu. & Max. \\
\hline \multirow{2}{*}{ UNMANAGED } & CAR speed & 0 & 26 & 48 & 39 & 50 & 60 \\
& CAR acc & 0 & 0.2 & 0.2 & 0.5 & 0.5 & 3.4 \\
& HDV speed & 0 & 36 & 48 & 41 & 49 & 57 \\
& HDV acc & 0.01 & 0.13 & 0.15 & 0.42 & 0.6 & 1.98 \\
& CAR speed & 0 & 33 & 48 & 40 & 50 & 60 \\
& CAR acc & 0 & 0.2 & 0.2 & 0.5 & 0.5 & 3.4 \\
& HDV speed & 19 & 47 & 49 & 48 & 50 & 58 \\
& HDV acc & 0.01 & 0.13 & 0.13 & 0.18 & 0.15 & 1.63 \\
\hline
\end{tabular}

The cumulative fuel economy and distance travelled are shown in Figure 5, for all three scenarios and vehicle fleet compositions

We can see that managed lane and ML+ACC greatly reduce truck fuel consumption (resp. 25\% and 40\% savings). Furthermore, light-duty vehicles are not negatively affected. The fuel consumption is significantly improved for heavy-duty trucks as a result of managed lane strategy. However, increased percentage of the HEV vehicles in traffic flow leads to negative impacts under the managed lane + ACC scenario. This is due to the fact, that the drive cycles are smooth and there is no regeneration. On the other hand a heavy battery increases the mass of the vehicles and leads to increase in fuel 
consumption. On the other hand, lane management scenario does not have any significant impact on fuel consumed by heavy duty vehicles. Removing, slowly moving trucks from the flow improves it and compensates the reduction of capacity. Since the light duty vehicle mostly flow in a stop-and-go traffic, level of hybridisation has a positive effect of fuel consumption for this calls of vehicles. The tabulated results for fuel savings are shown in Table 4.

Figure 5 Total fuel consumption for all nine scenarios for both light duty and heavy duty vehicles (see online version for colours)

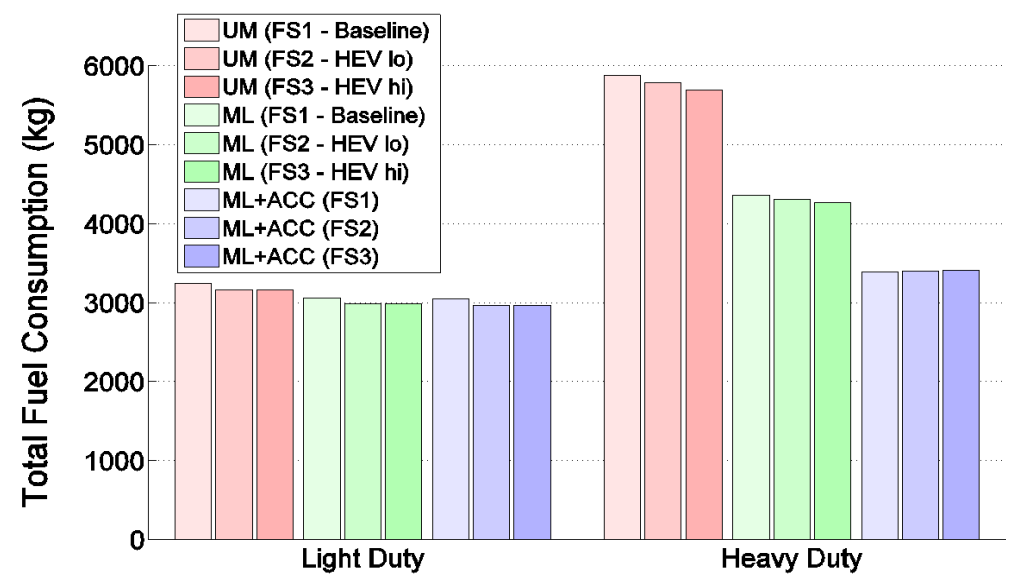

Table 4 Total fuel savings

\begin{tabular}{lccc}
\hline Fuel economy $(\mathrm{km} / \mathrm{l})$ & Unmanaged & Managed & Managed with $A C C$ \\
\hline Total & 6.21 & $6.69(+7.8 \%)$ & $7.32(+17.9 \%)$ \\
Car & 9.04 & $8.711(-3.7 \%)$ & $8.72(-3.6 \%)$ \\
HDV & 2.15 & $2.85(+32.6 \%)$ & $3.62(68.4 \%)$ \\
\hline
\end{tabular}

\section{Regional TMC implementation case study}

To evaluate the effectiveness and efficiency of the POLARIS ABM in simulating ITS response, an additional case study was conducted for the Chicago metropolitan area. In this case study our model was used to analyse the benefit of a simple ITS infrastructure in terms of the improved network performance and the adapted travel demand. In this section we present the setup of the case study and the results that have been obtained. The simulated area is an area surrounding the Chicago CBD, which has around 5,000 links, 3,000 intersections, 6,500 activity locations and 400,000 travellers. The simulated ITS infrastructure includes variable message sign (VMS) and highway advisory radio (HAR). Three scenarios were studied, including a normal day scenario, a scenario with incidents but with the ITS infrastructure disabled, and a scenario with incidents and the ITS infrastructure enabled. The incidents in the case study include multiple synthetic accident events on the major expressways and a heavy snow in the Chicago CBD region from 5am to noon. The impact of the accident and weather events to the network traffic was modelled by the reduction of link capacity and free-flow speed per the rules introduced in 
the FHWA guidebook. The ITS responses include notifications about accident events displayed on VMS located along the expressways and a continuously broadcasted weather advisory via the HAR.

Here we show the average network loading as a function of time and how it compares amongst the three scenarios. First, the average day instantaneous network load is plotted as a range of one standard deviation around the simulated mean. This is compared to the no-ITS and ITS scenarios with network events. The results in Figure 6 show the impact that the network events have on the network performance, with many more vehicles in the network at a given time than on an average day, indicating congestion and delay due to the events. The case with ITS responses shows the impact these have on the system performance, with the network load generally decreasing when vehicles are informed of events occurring. The provision of the accident/weather information allows the vehicle to reroute prior to experiencing the congestion, thereby reducing the load on the network. Overall, the use of ITS saves 30,000 hours of delay which is a reduction of $23 \%$ of the excess delay caused by the network events. It is important to note here that this case study is intended solely to demonstrate the capabilities of the ABM modelling system.

Figure 6 TMC case study network load results (see online version for colours)

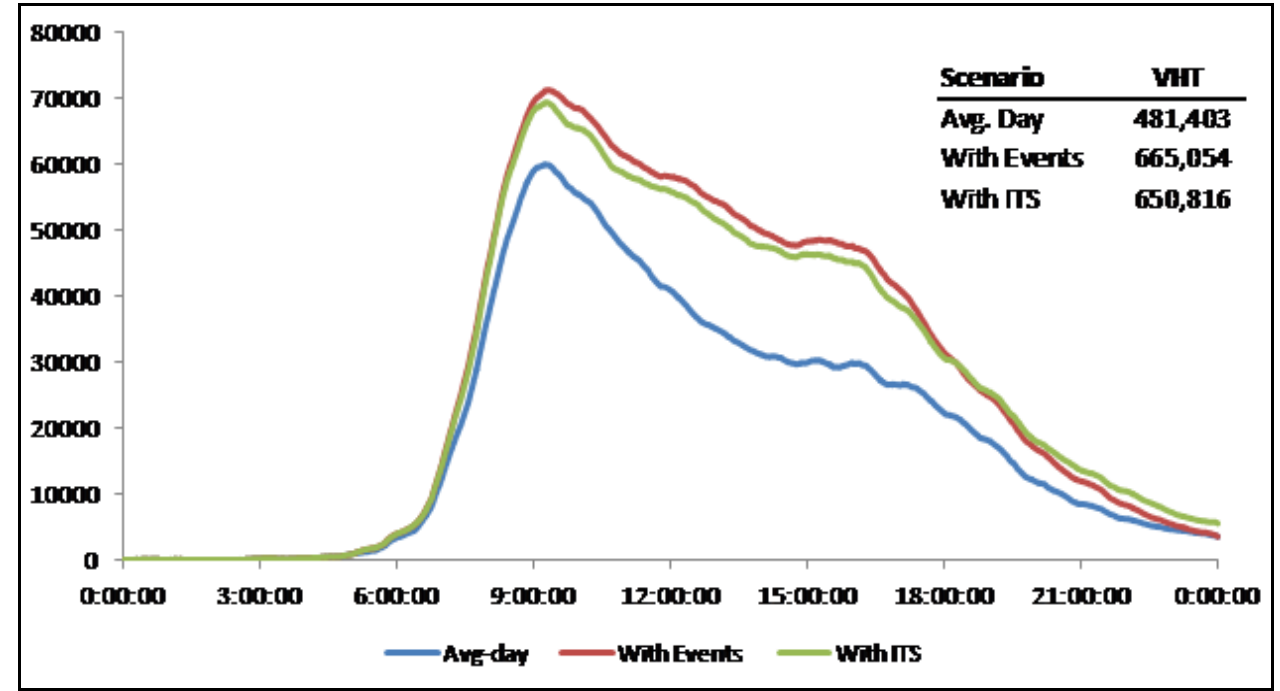

\section{Conclusions}

This paper demonstrates how a powertrain simulation software that is usually applied to study a given vehicle technology on a predefined drive cycle can be used to study realistic complex transportation systems. We demonstrated that an energy impact associated with adaptive cruise control system installed on trucks and managed lane used by trucks can be estimated though simulation. We believe that energy should be one of the metrics that is applied to studying ITS technologies by looking at each individual vehicle in a context of a complex system with other vehicles, traffic signals and congested road network. We demonstrated that it is possible to study impacts of ITS 
technologies on transportation systems not only by using aggregate measures such as vehicle miles travelled but by doing more precise microscopic simulation, which is sensitive to many parameters that cannot be represented in a framework that relies on VMT. Next steps in this work will focus on regional assessment of energy impacts from ITS technology deployment, as in the TMC case study above.

\section{Acknowledgements}

Work by the authors was supported by the Argonne National Laboratory's Laboratory Directed Research and Development.

\section{References}

Abou-Senna, H. and Radwan, E. (2013) 'VISSIM/MOVES integration to investigate the effect of major key parameters on CO2 emissions', Transportation Research Part D: Transport and Environment, Vol. 21, No. 0, pp.39-46, Univ Central Florida.

Advanced Light-Duty Powertrain and Hybrid Analysis (ALPHA) Tool, US EPA [online] https://www3.epa.gov/otaq/climate/alpha.htm (accessed 05/11/2015).

Armstrong, J.M. and Khan, A.M (2004) 'Modelling urban transportation emissions: role of GIS', Computers, Environment and Urban Systems, Vol. 28, No. 4, pp.421-433.

Auld, J. and Mohammadian, A. (2009) 'Framework for the development of the agent-based dynamic activity planning and travel scheduling (ADAPTS) model', Transportation Letters, Vol. 1, No. 3, pp.245-255.

Auld, J. and Mohammadian, A.K. (2012) 'Activity planning processes in the agent-based dynamic activity planning and travel scheduling (ADAPTS) model', Transportation Research Part A: Policy and Practice, Vol. 46, No. 8, pp.1386-1403.

Auld, J., Rashidi, T.H., Javanmardi, M. and Mohammadian, A.K. (2011) 'Dynamic activity generation model using competing hazard formulation', Transportation Research Record: Journal of the Transportation Research Board, Vol. 2254, No. 1, pp.28-35.

Autonomie, Argonne National Laboratory [online] http://www.autonomie.net/ (accessed 05/11/2015).

AVL CRUISE [online] https://www.avl.com/cruise/ (accessed 05/11/2015).

Babin, A., Florian, M., James-Lefebvre, L. and Spiess, H. (1981) 'Emme/2 an interactive graphic method for road and transit planning', RTAC Annual Conference Preprints, Vol. 1.

Balmer, M., Meister, K., Rieser, M., Nagel, K. and Axhausen, K.W. (2008) Agent-based Simulation of Travel Demand: Structure and Computational Performance of MATSim-T, ETH, Eidgenossische Technische Hochschule Zurich, IVT Institut fur Verkehrsplanung und Transportsysteme.

Barth, M., An, F., Norbeck, J. and Ross, M. (1996) 'Modal emissions modeling: a physical approach', Transportation Research Record: Journal of the Transportation Research Board, Vol. 1520, pp.81-88.

Ben-Akiva, M., Bierlaire, M., Koutsopoulos, H.N. and Mishalani, R. (2002) 'Real time simulation of traffic demand-supply interactions within DynaMIT', Transportation and Network Analysis: Current Trends, pp.19-36, Springer.

Borge, R., de Miguel, I., de la Paz, D., Lumbreras, J., Perez, J. and Rodriguez, E. (2012) 'Comparison of road traffic emission models in Madrid (Spain)', Atmospheric Environment, December, Vol. 62, pp.461-471, UPM, Madrid. 
Boriboonsomsin, K. and Barth, M. (2008) 'Impacts of freeway high-occupancy vehicle lane con.guration on vehicle emissions', Transportation Research Part D: Transport and Environment, Vol. 13, No. 2, pp.112-125, UC Riverside.

Brooker, A., Gonder, J., Wang, L., Wood, E., Lopp, S. and Ramroth, L. (2015) Fastsim: A Model to Estimate Vehicle Efficiency, Cost and Performance, Technical report, SAE Technical Paper.

California Air Resource Board (2011) EMFAC2011 Technical Documentation, CARB, California, USA.

Ciesla, C., Keribar, R. and Morel, T. (2000) Engine/Powertrain/Vehicle Modeling Tool Applicable to All Stages of the Design Process, Technical report, SAE Technical Paper.

Copert 4, Emisia SA [online] http://emisia.com/copert (accessed 05/11/2015).

Greenhouse Gas Emissions Model (GEM), US EPA [online] https://www3.epa.gov/otaq/ climate/gem.htm (accessed 05/11/2015).

GT-Suite Overview, Gamma Technologies [online] http://www.gtisoft.com/products/GTSUITE_Overview.php (accessed 05/11/2015).

Hatzopoulou, M. and Miller, E.J. (2010) 'Linking an activity-based travel demand model with traffic emission and dispersion models: transport's contribution to air pollution in Toronto', Transportation Research Part D: Transport and Environment, Vol. 15, No. 6, pp.315-325.

Hausberger, S., Rexeis, M., Zallinger, M. and Luz, R. (2009) Emission factors from the model PHEM for the HBEFA version 3, Report Nr. I-20/2009, Haus-Em 33/08/679.

Hirschmann, K., Zallinger, M., Fellendorf, M. and Hausberger, S. (2010) 'A new method to calculate emissions with simulated traffic conditions', 2010 13th International IEEE Conference on Intelligent Transportation Systems (ITSC), pp.33-38, IEEE.

Hülsmann, F., Gerike, R. and Ketzel, M. (2014) 'Modelling traffic and air pollution in an integrated approach - the case of Munich', Urban Climate, Vol. 10, pp.732-744.

Jayakrishnan, R., Mahmassani, H.S. and Hu, T-Y. (1994) 'An evaluation tool for advanced traffic information and management systems in urban networks', Transportation Research Part C: Emerging Technologies, Vol. 2, No. 3, pp.129-147.

Kwak, J., Park, B. and Lee, J. (2012) 'Evaluating the impacts of urban corridor traffic signal optimization on vehicle emissions and fuel consumption', Transportation Planning and Technology, Vol. 35, No. 2, pp.145-160.

Lawe, S., Bradley, M., Bowman, J.L., Roden, D.B., Castiglione, J. and Outwater, M.L. (2011) 'Design of an integrated, advanced travel demand model with a fine-grained, time-dependent network for a small urban area', 90th Annual Meeting of the Transportation Research Board, Washington, DC.

Lee, G., You, S., Ritchie, S., Saphores, J-D., Sangkapichai, M. and Jayakrishnan, R. (2009) 'Environmental impacts of a major freight corridor: a study of i-710 in California', Transportation Research Record: Journal of the Transportation Research Board, Vol. 2123, pp.119-128.

Lighthill, M.J. and Whitham, G.B. (1955) 'On kinematic waves. II. A theory of traffic flow on long crowded roads', Proceedings of the Royal Society of London: A Mathematical, Physical and Engineering Sciences, Vol. 229, pp.317-345, The Royal Society.

Ligterink, N.E. and de Lange, R. (2009) 'Refined vehicle and driving-behaviour dependencies in the VERSIT+ emission model', ETAPP Symposium.

Lu, C-C., Zhou, X. and Zhang, K. (2013) 'Dynamic origin-destination demand flow estimation under congested traffic conditions', Transportation Research Part C: Emerging Technologies, Vol. 34, pp.16-37.

Madireddy, M., de Coensel, B., Can, A., Degraeuwe, B., Beusen, B., de Vlieger, I. and Botteldooren, D. (2011) 'Assessment of the impact of speed limit reduction and traffic signal coordination on vehicle emissions using an integrated approach', Transportation Research Part D: Transport and Environment, Vol. 16, No. 7, pp.504-508. 
Mahmassani, H.S. and Stephan, D.G. (1988) Experimental Investigation of Route and Departure Time Choice Dynamics of Urban Commuters, Transportation Research Record Vol. 1203, pp.69-84.

Mahmassani, H.S., Peeta, S., Hu, T-Y. and Ziliaskopoulos, A. (1993) 'Dynamic traffic assignment with multiple user classes for real-time ATIS/ATMS applications', Large Urban Systems, Proceedings of the Advanced Traffic Management Conference.

Misra, A., Roorda, M.J. and MacLean, H.L. (2013) 'An integrated modelling approach to estimate urban traffic emissions’, Atmospheric Environment, Vol. 73, pp.81-91.

Nam, E. (2004) Advanced Technology Vehicle Modeling in Pere, Office of Transportation and Air Quality [online] https://www3.epa.gov/otaq/models/ngm/420d04002.pdf (accessed $11 / 5 / 2015)$.

Newell, G.F. (1993) 'A simplified theory of kinematic waves in highway traffic, Part I: General theory', Transportation Research Part B: Methodological, Vol. 27, No. 4, pp.281-287.

Ntziachristos, L., Gkatzoflias, D., Kouridis, C. and Samaras, Z. (2009) 'COPERT: a European road transport emission inventory model', Environmental Science and Engineering, pp.491-504, Aristotle Univ. Thessaloniki, Springer, Berlin Heidelberg.

Pendyala, R.M. (2014) Low Emissions Zones: Simulation of Behavioral Impacts, September, Arizona State Univ. [online] http://ntl.bts.gov/lib/54000/54900/54931/Low_Emissions Zones_Modeling_Report_-_Final_508_-_012715.pdf (accessed 11/5/2015).

Pendyala, R.M., Konduri, K.C., Chiu, Y-C., Hickman, M., Noh, H., Waddell, P., Wang, L., You, D. and Gardner, B. (2012) 'Integrated land use-transport model system with dynamic time-dependent activity-travel microsimulation', Transportation Research Record: Journal of the Transportation Research Board, Vol. 2303, No. 1, pp.19-27.

PTV Group (n.d.) VISSIM [online] http://vision-traffic.ptvgroup.com/en-us/products/ptv-vissim/ (accessed 11/5/2016).

Rakha, H.A., Ahn, K., Moran, K., Saerens, B. and van den Bulck, E. (2011) 'Virginia Tech comprehensive power-based fuel consumption model: model development and testing', Transportation Research Part D: Transport and Environment, Vol. 16, No. 7, pp.492-503.

Richards, P.I. (1956) 'Shock waves on the highway', Operations Research, Vol. 4, No. 1, pp.42-51.

Smit, R., Smokers, R. and Rabé, E. (2007) 'A new modelling approach for road traffic emissions: VERSIT+', Transportation Research Part D: Transport and Environment, Vol. 12, No. 6, pp.414-422.

Sokolov, V., Karbowski, D. and Kim, N. (2014) Assessing the Energy Impact of Traffic Management and ITS Technologies, September, ANL, 25th ITS Annual Meeting.

Stevanovic, A., Stevanovic, J., Zhang, K. and Batterman, S. (2009) 'Optimizing traffic control to reduce fuel consumption and vehicular emissions: integrated approach with VISSIM, CMEM, and VISGAOST', Transportation Research Record: Journal of the Transportation Research Board, Vol. 2128, pp.105-113.

US EPA (2014) MOVES2014a User Guide [online] https://www3.epa.gov/otaq/models/moves/ documents/420b15095.pdf.

Xie, Y., Chowdhury, M., Bhavsar, P. and Zhou, Y. (2012) 'An integrated modeling approach for facilitating emission estimations of alternative fueled vehicles', Transportation Research Part D: Transport and Environment, January, Vol. 17, No. 1, pp.15-20, Univ Mass Lowell.

Yang, Q., Koutsopoulos, H.N. and Ben-Akiva, M.E. (2000) 'Simulation laboratory for evaluating dynamic traffic management systems', Transportation Research Record: Journal of the Transportation Research Board, Vol. 1710, No. 1, pp.122-130.

Zachariadis, T. and Samaras, Z. (1999) 'An integrated modeling system for the estimation of motor vehicle emissions', Journal of the Air \& Waste Management Association, September, Vol. 49, No. 9, pp.1010-1026. 